\title{
MULTIPLICITY DISTRIBUTION IN HIGH ENERGY COLLISIONS *
}

\author{
Yukio TOMOZAWA \\ Stanford Linear Accelerator Center, Stanford University, Stanford, California 94305 and \\ Randall Laboratory of Physics, The University of Michigan \\ Ann Arbor, Michigan $48104^{\dagger}$
}

Received 9 April 1973

Abstract: It is shown that the asymptotic multiplicity distribution approaches a Gaussian distribution. The agreement with the experimental data indicates that the higher correlations should not be so strong.

Charged multiplicity distribution data are easily obtained in high energy collision experiments and have been discussed extensively in the literature. The existence of correlations in the pp collision data up to $300 \mathrm{GeV}$ clearly excludes the Poisson distribution, the simplest possible distribution. Nevertheless, the data shows a simple form for the multiplicity distribution and suggests that there is some regularity behind it [1]. In this note, we show that the form of the asymptotic distribution is Gaussian, and that it indicates a weak correlation among the produced particles.

The basis of the analysis is a theorem $[2,3]$ which states that if the higher correlations are not too strong (the precise meaning is specified later ${ }^{* *}$ ) the asymptotic distribution approaches the normal (Gaussian) distribution. This is an analogue of the central limit theorem in statistics and probability theory [5]. More explicitly, the normalized charged multiplicity distribution can be expressed as [3]

$$
P_{n} \equiv \frac{\sigma_{n}}{\sigma_{\text {inel }}}=\frac{1}{\sqrt{2 \pi} \beta} \exp \left[-\frac{(n-m)^{2}}{2 \gamma^{2}}\right]\left\{1+\sum_{k=3}^{\infty} a_{k}\left(\frac{n-m}{\gamma}\right)^{k}\right\},
$$

where all parameters are determined in terms of the cumulants $\kappa_{r}$ or the correlation moments $f_{r}$ defined by [5]

$$
\sum_{n=0}^{\infty} \mathrm{e}^{i n t} P_{n}=\exp \left[\sum_{r=1}^{\infty} \frac{\kappa_{r}(i t)^{r}}{r !}\right]=\exp \left[\sum_{r=1}^{\infty} \frac{f_{r}\left(\mathrm{e}^{i t}-1\right)^{r}}{r !}\right],
$$

i.e.

\footnotetext{
* Work supported in part by the U.S. Atomic Energy Commission.

$\dagger$ Permanent address.

** The multiperipheral model or some field theoretical model satisfies the condition; see [4] and references quoted therein.
} 


$$
\begin{aligned}
& \beta=\sqrt{\kappa_{2}}\left(1+\frac{1}{12} \frac{\kappa_{3}^{2}}{\kappa_{2}^{3}}-\frac{1}{8} \frac{\kappa_{4}}{\kappa_{2}^{2}}+O\left(\frac{1}{\kappa_{2}^{2}}\right)\right), \\
& \gamma=\sqrt{\kappa_{2}}\left(1+\frac{1}{4} \frac{\kappa_{3}^{2}}{\kappa_{2}^{3}}-\frac{1}{4} \frac{\kappa_{4}}{\kappa_{2}^{2}}+\mathrm{O}\left(\frac{1}{\kappa_{2}^{2}}\right)\right), \\
& m=\kappa_{1}-\frac{1}{2} \frac{\kappa_{3}}{\kappa_{2}}+\mathrm{O}\left(\frac{1}{\kappa_{2}}\right), \\
& a_{3}=\mathrm{O}\left(\frac{1}{\sqrt{\kappa_{2}}}\right), \quad a_{4,6}=\mathrm{O}\left(\frac{1}{\kappa_{2}}\right), \\
& a_{3 l-4,3 l-2,3 l}=\mathrm{O}\left(\kappa_{2}^{-\frac{1}{2} l}\right), l \geq 3 .
\end{aligned}
$$

The cumulants, the correlation moments, and the dispersion moments $\overline{(n-\bar{n})^{k}}$ are related to each other by

$$
\begin{aligned}
& \kappa_{1}=f_{1}=\bar{n}, \quad \kappa_{2}=f_{1}+f_{2}=\overline{(n-\bar{n})^{2}}, \quad \kappa_{3}=f_{1}+3 f_{2}+f_{3}=\overline{(n-\bar{n})^{3}}, \\
& \kappa_{4}=f_{1}+7 f_{2}+6 f_{3}+f_{4}=\overline{(n-\bar{n})^{4}}-3\left(\overline{(n-\bar{n})^{2}}\right)^{2}, \text { etc. }
\end{aligned}
$$

The assumptions which lead to eqs. (1) and (3) are [2,3] that (a) $\kappa_{2} \rightarrow \infty$, (b) the ratio $\kappa_{l} / \kappa_{2}(l>2)$ are bounded [4], and (c) $\left|(n-\bar{n}) / \kappa_{2}\right|<\pi$.

At extremely high energy, therefore, we expect to have a Gaussian distribution

$$
P_{n} \underset{s \rightarrow \infty}{\longrightarrow} \frac{1}{\sqrt{2 \beta}} \exp \left[-\frac{(n-m)^{2}}{2 \gamma^{2}}\right] \text {, }
$$

with

$$
\begin{aligned}
& m \underset{s \rightarrow \infty}{\longrightarrow} \bar{n}-\frac{1}{2} \frac{\overline{\left(\overline{n-\bar{n})^{3}}\right.}}{\overline{(n-\bar{n})^{2}}}, \\
& \beta=\frac{1}{\sqrt{2 \pi}} \frac{\sigma_{\text {inel }}}{\sigma_{\mathrm{m}}} \underset{s \rightarrow \infty}{\longrightarrow} \sqrt{\overline{(n-\bar{n})^{2}}} \\
& \underset{s \rightarrow \infty}{\longrightarrow} \gamma,
\end{aligned}
$$

where $\sigma_{m}$ stands for the maximum value of the distribution function ${ }^{\dagger}$. However, it can be recognized immediately that the convergence to the limiting form, eqs. (5-8), is rather slow. The reason for this is that the normal distribution with $\beta=\gamma$ is normalized in the integral $(-\infty, \infty)$ and the integral over the unphysical internal $(-\infty, 0)$ is

$\dagger_{m}$ is called the modal multiplicity, and its usefulness is demonstrated in the analysis of Webber [6]. 


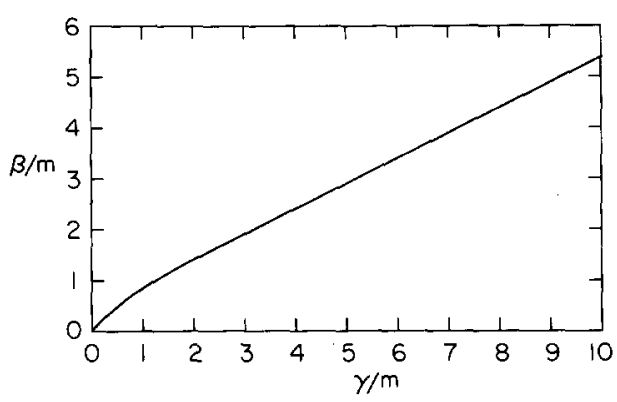

Fig. 1. The curve for $\beta / m=g(\gamma / m)$, eq. (10).

not negligible at the present energy. Moreover, the speed of the convergence of the parameters $\beta$ and $\gamma$ to the asymptotic value $\sqrt{(n-\bar{n})^{2}}$ is not the same as is seen from eq. (3).

In order to obtain a more effective formula with predictive power, we impose the normalization condition ${ }^{*}$ on eq. (5),

$$
1=\int_{0}^{\infty} P_{n} \mathrm{~d} n=\frac{\gamma}{\sqrt{2 \pi \beta}} \int_{-m / \gamma}^{\infty} \mathrm{e}^{-t^{2} / 2} \mathrm{~d} t,
$$

i.e.

$$
\frac{\beta}{m}=g(\gamma / m)=\frac{1}{2} \frac{\gamma}{m}\left[1+\operatorname{Erf}\left(\frac{m}{\sqrt{2} \gamma}\right)\right] .
$$

The function $g(x)$ is depicted in fig. 1 . It is easily observed that

$$
1<\gamma / \beta<2,
$$

where the lower (upper) bound corresponds to the limit $\gamma / m \rightarrow O(\infty)$.

Assuming the asymptotic form (5) and the condition (10), we analyze the experimental data with the following procedure:

(i) Consider the negative charged multiplicity, $n_{-}=\frac{1}{2} n_{\mathrm{ch}}-1$.

(ii) Find the modal multiplicity $m_{-}$and the maximum cross section $\sigma_{m_{-}}$from the three largest cross section using the Gaussian form. If the middle point of the three corresponds to the largest, as is the case for $E_{\mathrm{L}} \geq 200 \mathrm{GeV}$, the parabola approximation is good enough. (We avoid using the $n_{-}=0$ point because of an ambiguity due to the elastic cross section.) Disregard the $\gamma$ obtained here.

(iii) Determine $\beta$ by eq. (7) and $\gamma$ by eq. (10) or fig. 1 .

(iv) Plot $\log _{10}\left(\sigma_{n_{-}} / \sigma_{m_{-}}\right)$against $\log _{10} \mathrm{e}\left(n_{-}-m_{-}\right)^{2} / 2 \gamma^{2}$.

* After completion of the work, the author came across the works of ref. [7], in which a similar analysis was made. The author believes, however, that the treatment of the present article is more transparent in presening the reasoning of using the Gaussian form, the analysis of the experimental data, and suggesting a possible correction term. 


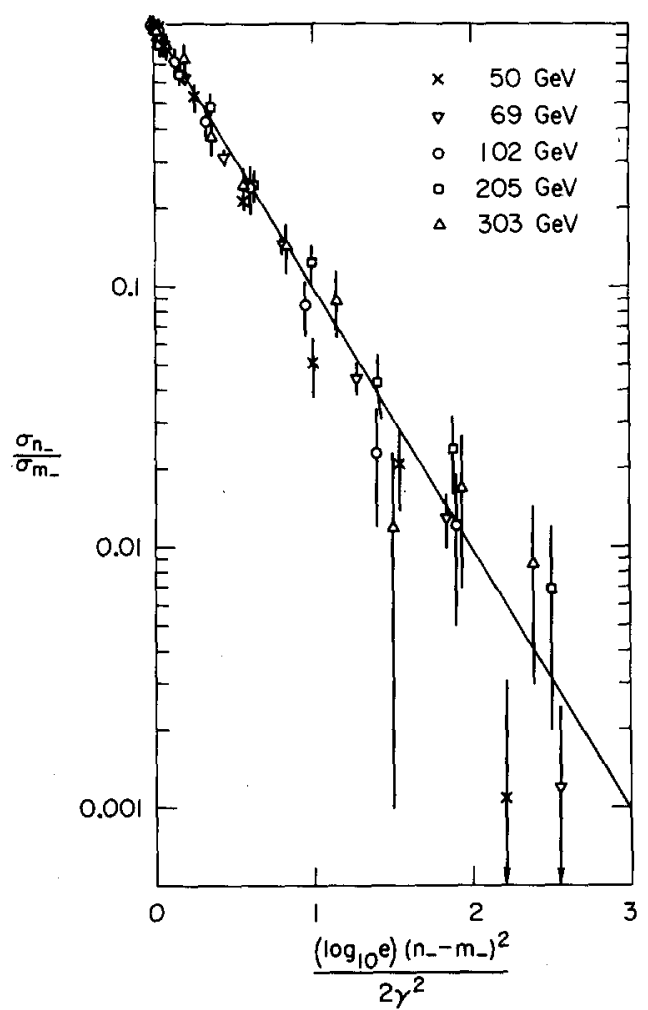

Fig. 2. The negative charge multiplicity distribution. The solid line represents the expected asymptotic limit.

(v) The data points should approach the asymptotic limit, the straight line with the gradient -1 .

The last step may be replaced by:

$\left(v^{\prime}\right)$ Plot $\log _{10}\left(\sigma_{n_{-}} / \sigma_{m_{-}}\right)$against $\left(n_{-}-m_{-}\right)^{2}$. Determine $\gamma^{2}$ by the gradient of an expected straight line and compare the result with that obtained in step 4.

The result of the analysis based on the procedures (i)-(v) is shown in fig. 2 and table 1. Some discussion is in order.

(a) While the data points for $50 \mathrm{GeV}$ given in fig. 2 are slightly off the expected asymptotic line, those for $69-300 \mathrm{GeV}$ fall quite well on it. Even for $50 \mathrm{GeV}$, the data falls on a straight line. Thus, a slight change of the slope parameter $\gamma$ within its experimental error seems to restore the agreement between the data and prediction.

(b) The asymptotic relation (7) is well satisfied already at the present energy, while the others, eqs. (6) and (8) are not. In order to understand the difference in the speed of convergence, we estimate the parameters $\beta$ and $\gamma$ using eq. (3) and the experimental 
Table 1

The values for the parameters

\begin{tabular}{llllll}
\hline$E(\mathrm{GeV})$ & 50 & 69 & 102 & 205 & 303 \\
\hline$m_{-}$ & 0.96 & 1.19 & 1.21 & 2.0 & 2.24 \\
$\beta$ & $1.32 \pm 0.12$ & $1.44 \pm 0.06$ & $1.59 \pm 0.15$ & $1.88 \pm 0.15$ & $2.21 \pm 0.20$ \\
$\gamma$ & $1.90 \pm 0.17$ & $1.99 \pm 0.08$ & $2.26 \pm 0.22$ & $2.35 \pm 0.19$ & $2.90 \pm 0.26$ \\
$\kappa_{1}=f_{1}$ & $1.66 \pm 0.07$ & $1.95 \pm 0.04$ & $2.17 \pm 0.07$ & $2.82 \pm 0.08$ & $3.43 \pm 0.10$ \\
$\kappa_{2}=\frac{(n-\bar{n})^{2}}{(n-\bar{n})^{3}}$ & $1.67 \pm 0.11$ & $2.10 \pm 0.07$ & $2.56 \pm 0.12$ & $3.77 \pm 0.22$ & $4.80 \pm 0.29$ \\
$\kappa_{3}=0.32$ & $2.07 \pm 0.21$ & $2.60 \pm 1.31$ & $5.24 \pm 0.99$ & $7.07 \pm 1.60$ \\
\hline$\beta / \sqrt{\kappa_{2}}$ & $1.39 \pm 0.07$ & & & \\
$\gamma / \beta$ & $1.02 \pm 0.12$ & $0.99 \pm 0.05$ & $1.00 \pm 0.13$ & $0.97 \pm 0.11$ & $1.01 \pm 0.12$ \\
$\gamma / m$ & $1.44 \pm 0.13$ & $1.38 \pm 0.06$ & $1.42 \pm 0.13$ & $1.25 \pm 0.12$ & $1.31 \pm 0.12$ \\
$\left(\kappa_{3} / 2 \kappa_{2}\right)\left(\frac{1}{\bar{n}-m}\right)$ & $1.98 \pm 0.12$ & $1.67 \pm 0.07$ & $1.87 \pm 0.18$ & $1.18 \pm 0.10$ & $1.29 \pm 0.12$ \\
\hline & $0.60 \pm 0.23$ & $0.64 \pm 0.13$ & $0.53 \pm 0.33$ & $0.84 \pm 0.29$ & $0.61 \pm 0.18$ \\
\hline
\end{tabular}

data for the cumulants. At $303 \mathrm{GeV}$, we obtain

$$
\begin{aligned}
& \frac{\beta}{\sqrt{\kappa_{2}}}=1+(0.005 \pm 0.03), \\
& \frac{\gamma}{\sqrt{\kappa_{2}}}=1+(0.05 \pm 0.08) .
\end{aligned}
$$

This is consistent with the values given in table 1 .

(c) The relation (7) is asymptotically equivalent to that obtained by Weisberger [8], $\sqrt{\kappa_{2}} P_{\bar{n}}=1 / \sqrt{2 \pi}$ although the convergence to (7) seems faster.

(d) The experimental values for $(\gamma / m, \beta / m)$ are moving down on the curve of fig. 1 as energy increases. This seems to indicate that the data points are moving towards the limit $\gamma / m \rightarrow 0$ and $\gamma / \beta \rightarrow 1$. (This case may be called the weak two-body correlation model [4].)

(e) However, the possiblity of having the condition

$$
\frac{\gamma}{m} \underset{s \rightarrow \infty}{\longrightarrow} a \neq 0
$$

is not excluded. If that is the case, we obtain the KNO scaling law [9] with the Gaussian scaling function,

$$
m P_{n}=\frac{1}{\sqrt{2 \pi} g(a)} \exp \left[-\frac{1}{2 a}\left(\frac{n}{m}-1\right)^{2}\right] \text {. }
$$

(f) The step (v) is preferable to ( $\left.v^{\prime}\right)$ since the asymptotic form (1) or (5) is the best 
approximation around the modal point. Besides, the predictive power is more evident if step ( $v$ ) is used. If the accuracy of experimental data at higher energies is improved, we may be able to detect the polynomial term which we have neglected. In particular, the $a_{3}$ term, which is of the order $\mathrm{O}\left(1 / \sqrt{\kappa_{2}}\right)$, may be detected from the asymmetry of the curve. (The present accuracy does not permit us to detect such asymmetry.)

The author is indebted to Professors W.K.H. Panofsly and S.D. Drell for their hospitality at SLAC. Thanks are also due to many people at SLAC for useful suggestions and computation assistance; in particular, S. Brodsky, R. Cahn M.S. Chen, M. Kugler and Y.S. Tsai, to name a few. He is grateful to S. Brodsky and M. Chanowitz for reading the manuscript.

\section{References}

[1] V.V. Ammosov et al., Phys. Letters 42B (1972) 519;

J.W. Chapman et al., Phys. Rev. Letters 29 (1972) 1686;

G. Charlton et al., Phys. Rev. Letters 29 (1972) 515;

F.T. Dao, Phys. Rev. Letters 29 (1972) 1627.

[2] J.B.S. Haldane, Biometrika 32 (1942) 294.

[3] Y. Tomozawa, Asymptotic multiplicity distribution and analogue of the central limit theorem, Phys. Rev. D, to be published.

[4] W. Frazer et al., Rev. Mod. Phys. 44 (1972) 284.

[5] M.G. Kendall and A. Stuart, The advanced theory of statistics, vol. 1 (Griffin, London, 1963).

[6] B.R. Webber, Phys. Letters 42B (1972) 69.

[7] G.D. Kaiser, Nucl. Phys. B44 (1972) 171;

G.W. Parry and P. Rotelli, Application of the truncated Gaussian to the inelastic pp charged multiplicity distribution, Trieste preprint $\mathrm{IC} / 73 / 3$.

[8] W.I. Weisberger, Asymptotic multiplicity distributions in high energy collisions, Stony Brook preprint (1973).

[9] Z. Koba, H.B. Nielsen and P. Olesen, Nircl. Phys. B40 (1972) 317. 